\title{
The Revised Arthropod Containment Guidelines
}

\author{
Stephen Higgs
}

$\mathbf{I}_{B}^{\mathrm{N}}$ N 2003, JUST A few months after I became editor of VectorBorne and Zoonotic Diseases, we published the Arthropod Containment Guidelines, likely our first of what would become known as an Open Access publication. The concept to produce the guidelines resulted from a discussion with staff from the National Institutes of Health that had been involved in supporting efforts to develop the capacity to genetically engineer mosquitoes. What followed was more than a 2-year effort by members of the American Committee for Medical Entomology (ACME) - a subgroup of the American Society of Tropical Medicine and Hygiene (ASTMH). Drafts of the document were widely circulated among researchers and vector biologists, and revised several times until a consensus document was finalized. The guidelines were rapidly embraced by the international community as the "go-to" reference document for those working with arthropod vectors in laboratories, and administrators on committees that oversee these facilities.

The guidelines did not address fieldwork, including the use of human subjects, in part because some members/contributors did not want these topics covered without further discussion. This exclusion was subsequently discussed in another publication by Vector-Borne and Zoonotic Diseases (Achee et al. 2015). As a halfway point between laboratory and the field, and in the context of evaluating genetically engineered mosquitoes, the design and operation of field cages to evaluate engineered mosquitoes was discussed by Benedict et al. (2008) and Higgs (2008). The publication describing caged field trials were a response to the improved technologies related to genetic manipulation of mosquitoes, primarily in efforts to control malaria and dengue. This publication was also relevant to the need to evaluate sterile insect methods as described by Alphey et al. (2010) and nongenetic manipulation strategies for population suppression or replacement, including those based on Wolbachia (Higgs 2013).

The technological progress since 1991 when I came to the United States, specifically to learn about arthropod genetic engineering, was slow but steady with, for example, the discovery and application of transposable elements such as PiggyBac (Fraser et al. 1983). I had been in multiple meetings for a 25-year period, listening to discussion on what seemed to be the elusive Holy Grail. Even before genetic-based strategies to disrupt pathogen transmission/incidence (desirable traits) were known, it was realized that to be successful, some method would be needed to facilitate dispersal into natural populations-that is, Gene Drive. The field cages described by Benedict et al. (2008) specifically addressed mosquitoes with the then, mythical gene drive.

Without warning and with no obvious fanfare gene drive arrived. In the past 3 years the rate of progress has accelerated as a result of the development of CRISPR/Cas9 for use as a gene drive system in invertebrates and vertebrates (Cong et al. 2013, Mali et al. 2013, Ma et al. 2014, Zhang et al. 2014). In a remarkably short period of time CRISPR/Cas9 and derivatives have been applied to a wide range of organisms. By the time that the group with which I worked had published their discussions (National Academies of Sciences 2016) applications of CRISPR/Cas9 to mosquitoes had already been published for Anopheles stephensi (Gantz et al. 2015), Anopheles gambiae (Hammond et al. 2016), and Aedes aegypti (Kistler et al. 2015). For A. gambiae, the technology has already been used to knockout a gene that reduced infection with malaria parasites (Dong et al. 2018). As readers of Vector-Borne and Zoonotic Diseases will surely know, the technology quickly became readily available, relatively simple, and inexpensive. The days when we thought that Drosophila was easy, a few species were feasible but most were impossible, are over. The premise that the technology was most useful, but not necessarily restricted to sexually reproducing organisms with short generation times, and would be guided by normally accepted, if not legal, ethical standards has been dramatically challenged with announcements of use in human embryos.

In 2018, the journal published three companion articles on gene-modified mosquitoes (Mumford et al. 2018, Quinlan et al. 2018a, 2018b) that included considerations of containment. Although technology has changed, the risk assessments described in the original ACG for vector arthropods containing recombinant DNA molecules have basically remained the same. In 2003, based on much discussion by experts it was concluded that transgenic mosquitoes could be safely contained at level two. The obvious power of CRISPR/Cas9-based gene drives and the need to prevent inadvertent release of modified arthropods before full

Biosecurity Research Institute (BRI), Kansas State University, Manhattan, Kansas. 
environmental risk assessment has stimulated refinements of the original ACG specific to gene drive research (Benedict et al. 2008, Adelman et al. 2017) and serve as complements to the original ACG.

The majority of research with arthropods of medical and veterinary importance, however, comprises field studies or experimental manipulations with pathogens. Two influential changes that affect such research have taken place since the original ACGs were published, at least in the United States: the Select Agent rule and expansion of institutional regulatory compliance, particularly biosafety. A third of the pathogens on the HHS Select Agent List (7CFR Part 331, 9 CFR Part 121, and 42 CFR Part 73) are vector borne and any laboratory engaged in vector-pathogen research, including one of these, is now subject to federal oversight. The everexpanding compliance requirements meant that ACG was increasingly looked to as the basis for ensuring biosafety and, in many cases, comprised a checklist approach by biosafety officers. The Select Agent rule was not in place when the ACG was published. And, the ACG was never intended to be proscriptive. Anecdotally, researchers were increasingly burdened by new requirements to conduct their research, particularly with respect to infrastructure, and some were hobbled by the "ACG requirements." It became apparent that it was time to update the ACG to reflect these and other issues.

As with any such guideline document related to scientific fields that are changing, some frequency of revision is inevitably necessary and the document must evolve with the field. Appendix III of these new ACGs summarizes the changes. It is reassuring that new expertise and review by some of the original authors of the first ACGs have basically left the original ACG intact, but refined and updated the guidelines based on experience working under the original document. Language has been strengthened to emphasize that as with the original ACG, researchers and administrators should not read and interpret these recommendations simply from a perspective of a checklist-based approach but rather, reach their decisions on a case-by-case biosafety risk assessment. Local risk assessments can allow for alternative ways of mitigating risk if a laboratory cannot fully integrate the recommendations of the ACG. The new version is fully in agreement with the recent rethinking of biosafety by the World Health Organization, which has been concerned that the perceived "need" for expensive containment facilities in resource-poor sites may hinder scientific and public health progress; the new WHO Laboratory Biosafety Manual emphasizes practices and procedures, not engineering controls (Kojima et al. 2018).

If you really want to see how far we have come, you can go to the very first issue of Vector-Borne and Zoonotic Diseases in 2001 and read the review "Issues in Public Heath Entomology," the very first Vector-Borne and Zoonotic Diseases article ever, by Spielman et al. (2001). For those readers who knew Andy, he was never afraid to express his opinion and to approach issues face-on. The article discusses genetic manipulation of vectors and their potential release, mosquitoes and arboviruses, ticks and Lyme disease, persistent insecticides, introduction and spread of foreign arthropods, the Roll Back Malaria program, legal ramifications of vector-borne diseases, ethical concerns, the inhibitory effect that biosafety recommendations can have on research, education and what was then called, global warming rather than climate change. Most of these issues remain on the front burner. In anticipation of the updated ACG, Andy and colleagues wrote as follows: "Effective biosafety may often be based solely on appropriate practices and procedures, guided by experience, without a need for specialized equipment or facilities."

\section{References}

Achee NL, Youngblood L, Bangs MJ, Lavery JV, et al. Considerations for the use of human participants in vector biology research: A tool for investigators and regulators. Vector Borne Zoonotic Dis 2015; 15:89-102.

Adelman ZN, Pledger D, Myles KM. Developing standard operating procedures for gene drive research in disease vector mosquitoes. Pathog Glob Health 2017; 111:436-447.

Alphey L, Benedict M, Bellini R, Clark GG, et al. Sterile-insect methods for control of mosquito-borne diseases: An analysis. Vector Borne Zoonotic Dis 2010; 10:295-311.

Benedict M, D'Abbs P, Dobson S, Gottlieb M, et al. Guidance for contained field trials of vector mosquitoes engineered to contain a gene drive system: Recommendations of a scientific working group. Vector Borne Zoonotic Dis 2008; 8:127-166.

Cong L, Ran FA, Cox D, Lin S, et al. Multiplex genome engineering using CRISPR/Cas systems. Science 2013; 339:819-823.

Dong Y, Simoes ML, Marois E, Dimopoulos G. CRISPR/Cas9mediated gene knockout of Anopheles gambiae FREP1 suppresses malaria parasite infection. PLoS Pathog 2018; 14: e1006898.

Fraser MJ, Smith GE, Summers MD. Acquisition of host cell DNA sequences by baculoviruses: Relationship between host DNA insertions and FP mutants of Autographa californica and Galleria mellonella nuclear polyhedrosis viruses. J Virol 1983; 47:287-300.

Gantz VM, Jasinskiene N, Tatarenkova O, Fazekas A, et al. Highly efficient Cas9-mediated gene drive for population modification of the malaria vector mosquito Anopheles stephensi. Proc Natl Acad Sci U S A 2015; 112:E6736-E6743.

Hammond A, Galizi R, Kyrou K, Simoni A, et al. A CRISPRCas9 gene drive system targeting female reproduction in the malaria mosquito vector Anopheles gambiae. Nat Biotechnol 2016; 34:78-83.

Higgs S. Editorial and commentary on the guidance document for the contained field trials of engineered mosquitoes. Vector Borne Zoonotic Dis 2008; 8:125-126.

Higgs S. Alternative approaches to control dengue and chikungunya: Transgenic mosquitoes. Public Health 2013; 24:35-42.

Kistler KE, Vosshall LB, Matthews BJ. Genome engineering with CRISPR-Cas9 in the mosquito Aedes aegypti. Cell Rep 2015; 11:51-60.

Kojima K, Booth CM, Summermatter K, Bennett A, et al. Riskbased reboot for global lab biosafety. Science 2018; 360:260262.

Ma Y, Zhang L, Huang X. Genome modification by CRISPR/Cas9. FEBS J 2014; 281:5186-5193.

Mali P, Yang L, Esvelt KM, Aach J, et al. RNA-guided human genome engineering via Cas9. Science 2013; 339:823-826.

Mumford JD, Leach AW, Benedict MQ, Facchinelli L, et al. Maintaining quality of candidate strains of transgenic mos- 
quitoes for studies in containment facilities in disease endemic countries. Vector Borne Zoonotic Dis 2018; 18:31-38. National Academies of Sciences, Engineering, and Medicine. Gene Drives on the Horizon: Advancing Science, Navigating Uncertainty, and Aligning Research with Public Values. Washington, DC: The National Academies Press, 2016.

Quinlan MM, Birungi J, Coulibaly MB, Diabate A, et al. Containment studies of transgenic mosquitoes in disease endemic countries: The broad concept of facilities readiness. Vector Borne Zoonotic Dis 2018a; 18:14-20.

Quinlan MM, Mutunga JM, Diabate A, Namountougou M, et al. Studies of transgenic mosquitoes in disease-endemic countries: Preparation of containment facilities. Vector Borne Zoonotic Dis 2018b; 18:21-30.
Spielman A, Pollack RJ, Kiszewski AE, Telford SR, III. Issues in public health entomology. Vector Borne Zoonotic Dis 2001; 1:3-19.

Zhang F, Wen Y, Guo X. CRISPR/Cas9 for genome editing: Progress, implications and challenges. Hum Mol Genet 2014; 23:R40-R46.

Address correspondence to:

Stephen Higgs

Biosecurity Research Institute (BRI)

Kansas State University Manhattan, KS 66506

E-mail: shiggs@k-state.edu 